\title{
Dynamically Corrected Transition State Theory Calculations of Self-Diffusion in Anisotropic Nanoporous Materials
}

\author{
D. Dubbeldam, $*, \dagger$ E. Beerdsen, $\pitchfork, \S$ S. Calero,,$*$ and B. Smit ${ }^{\dagger, \S}$ \\ Van't Hoff Institute for Molecular Sciences, University of Amsterdam, Nieuwe Achtergracht 166, 1018 WV \\ Amsterdam, The Netherlands, Department of Experimental Sciences, University Pablo de Olavide, Ctra. Utrera \\ km 1. 41013 Sevilla, Spain, and Cecam (European Centre for Atomic and Molecular Computations), Ecole \\ Normale Supérieure 46, Allée d'Italie 69364 Lyon, Cedex 7, France
}

Received: August 1, 2005; In Final Form: November 24, 2005

\begin{abstract}
We apply the dynamically corrected transition state theory to confinements with complex structures. This method is able to compute self-diffusion coefficients for adsorbate-adsorbent systems far beyond the time scales accessible to molecular dynamics. Two example cage/window-type confinements are examined: ethane in ERI- and CHA-type zeolites. In ERI-type zeolites, each hop in the $z$ direction is preceded by a hop in $x y$ direction and diffusion is anisotropic. The lattice for CHA-type zeolite is a rhombohedral Bravais lattice, and diffusion can be considered isotropic in practice. The anisotropic behavior of ERI-type cages reverses with loading, i.e., at low loading the diffusion in the $z$ direction is two times faster than in the $x y$ direction, while for higher loadings this changes to a $z$ diffusivity that is more than two times slower. At low loading the diffusion is impeded by the eight-ring windows, i.e., the exits out of the cage to the next, but at higher loadings the barrier is formed by the center of the cages.
\end{abstract}

\section{Introduction}

Transport of adsorbates in nanoporous adsorbents such as zeolites is determined by a complex interplay between adsorbentadsorbate and adsorbate-adsorbate interactions. From a scientific point of view, zeolites are ideal systems to study the effect of confinement on the properties of the adsorbed molecules because of their regularity and periodicity. Although interesting effects such as single-file diffusion, ${ }^{1-3}$ incommensurate diffusion, ${ }^{4-7}$ and levitation effects ${ }^{8}$ are well-known, most of the effects of confinement on diffusion remain poorly understood. This is particularly true for loading effects in materials with different channels and/or cages in the $x, y$, and $z$ direction.

Anisotropic single-component diffusion in nanoporous materials has been known for a long time. A well-known example is diffusion in silicalite. ${ }^{9-13}$ In general, the diffusion coefficients in the different directions can have different dependencies on temperature and loading. A limited number of studies deal with nonzero loading. Bussai et al. ${ }^{14}$ found little change in anisotropy for water in silicalite as a function of loading, while Skoulidas and Sholl found a more irregular loading dependence of anisotropy. ${ }^{15}$ Anisotropic behavior is very common in nanoporous materials. The papers of Skoulidas and Sholl ${ }^{16}$ (molecular dynamics simulations), Trinh et al. ${ }^{17}$ (Monte Carlo simulations), Su et al. ${ }^{18}$ (molecular dynamics simulations in clay), Powles et al. ${ }^{19}$ (a model for permeable micropores with variable anisotropic diffusion), Yokoyamaa and Nakashimab ${ }^{20}$ (diffusion experiments on a rhyolite rock having anisotropic pore structure), Wingen et al. ${ }^{21}$ (anisotropic motion of water in zeolites EMT, L, and ZSM-5 as studied by D- and H NMR line splitting),

\footnotetext{
* Corresponding author. E-mail: dubbelda@science.uva.nl.

$\dagger$ University of Amsterdam.

$\doteqdot$ University Pablo de Olavide.

$\S$ Cecam.
}

Nelson et al. ${ }^{23,22}$ (modeling permeation through anisotropic zeolite membranes), Furo and Dvinskikh ${ }^{24}$ (methodology of NMR experiments intended to measure anisotropic diffusion), and Manzel et al. ${ }^{25}$ (NMR characterization of the pore structure and anisotropic self-diffusion in saltwater ice) are just a few examples.

Although molecular dynamics (MD) is a very powerful technique to study these effects, MD is typically limited to diffusion rates on the order of $10^{-12} \mathrm{~m}^{2} / \mathrm{s}$. To overcome this, some studies have used dynamically corrected transition state theory (dcTST) methods (see refs 26-28 and references therein). Hitherto, studies were limited to the infinite dilution limit, whereas many of the processes of practical importance occur at nonzero loading. Beerdsen et al. ${ }^{29}$ resolved this problem by extending the dcTST Bennett-Chandler approach to include diffusion of molecules at nonzero loading using only assumptions already present in TST.

In this work, we report molecular simulations of diffusion in confinement showing a phenomenon previously denoted as molecular path control (MPC); depending on loading, molecules follow a preferred pathway. ${ }^{32}$ We note that MPC is different from molecular traffic control, ${ }^{33}$ which is caused by mutual correlations in the movement of a multicomponent fluid through two types of pores. As a specific MPC example, we study the mechanism behind tunable anisotropy of ethane in ERI-type zeolite membranes. In ERI-type zeolite, the diffusion is characterized by complex diffusion paths. Each hop in the $z$ direction is preceded by a hop in the $x y$ direction. At low loading, the diffusivity in the $z$ direction is two times faster than in the $x y$ direction for both the self- and collective diffusivity, while for higher loadings this changes into a $z$ diffusivity that is more than two times slower. Additionally, we study a closely related zeolite known as Chabazite (CHA). The cages of CHA-type zeolites are somewhat smaller than ERI-type cages, and more 


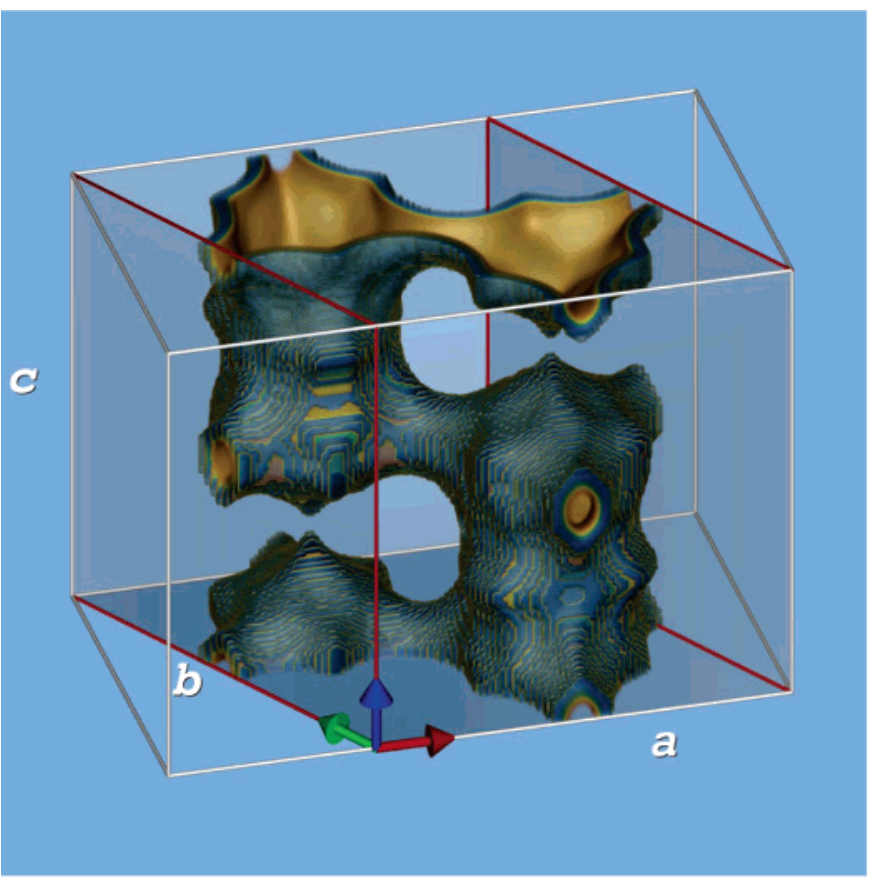

(a)

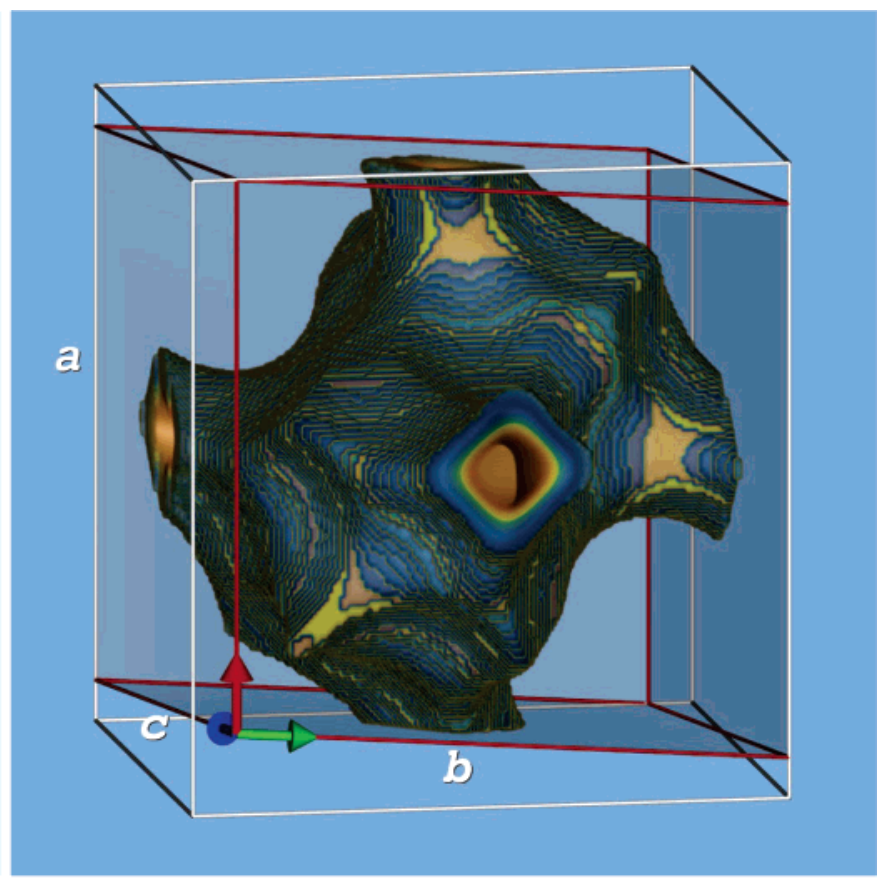

(b)

Figure 1. Unit cell of (a) ERI-type zeolite, and (b) CHA-type zeolite. The ERI-type structure ${ }^{30}$ crystallizes in the hexagonal dipyramidal space group $P 6_{3} / \mathrm{mmc}$ with $a=b=1.327 \mathrm{~nm}, c=1.505 \mathrm{~nm}$, and $\alpha=\beta=90^{\circ}, \gamma=120^{\circ}$. The CHA-type structure ${ }^{31}$ has the space group $R 3 \mathrm{~m}$ (a squashed cube) with $a=b=c=0.9459 \mathrm{~nm}$, and $\alpha=\beta=\gamma=94.07^{\circ}$. A unit cell of erionite contains two cages, while a unit cell of chabazite contains a single cage.

spherical. In literature, a rectangular hopping lattice has been used for CHA to compute diffusion in simulations. ${ }^{34,35}$ For this lattice the diffusion "appears" to be anisotropic. However, for a different lattice properly aligned with the crystal axes we show that in fact diffusion in CHA-type zeolites is nearly isotropic. We note that the problem in zeolites is reversed to lattice theory where the hopping rates and lattice-type are given. Here, we need to resolve both, and for this we apply the dcTST method. We use dcTST and MD to study changes in diffusion behavior in ERI- and CHA-type zeolites over loading.

The remainder of this paper is organized as follows. In section II we start with a detailed description of the ERI- and CHAtype zeolites. Next, we describe the dcTST method to compute the effective hopping rate for these lattices. In the results section III we derive the correct conversions from hopping rates to diffusivities, and show results for ethane at $600 \mathrm{~K}$ in ERI- and CHA-type zeolites using dcTST and MD. The free-energy profiles, the transmission coefficients, the hopping rate, and selfdiffusion coefficients for both zeolites are evaluated. Here, we explain our choice of the hopping lattice, being closely related to the computed free-energy profiles. We end with conclusions on anisotropic behavior in zeolites as a function of loading and explain why the dcTST method is a suitable method to provide detailed insight into mechanisms behind it.

\section{Methods}

A. Zeolite Descriptions. The ERI-type silica structure ${ }^{30}$ crystallizes in the hexagonal dipyramidal spacegroup $P 6_{3} / m m c$ with $a=b=1.327 \mathrm{~nm}, c=1.505 \mathrm{~nm}$, and $\alpha=\beta=90^{\circ}, \gamma=$ $120^{\circ}$. The elongated erionite cages approximate the shape of $1.3 \times 0.63 \mathrm{~nm}$ cylinders connected by $0.36 \times 0.51 \mathrm{~nm}$ windows. Only linear molecules are able to penetrate the eight-membered ring windows. There are three windows at the top of the cage rotated $120^{\circ}$ with respect to one another. At the bottom of the cage there are also three windows rotated $120^{\circ}$ with respect to one another. The top three windows are aligned with the windows at the bottom. Each ERI-type unit cell contains two erionite cages as shown in Figure 1a.

The CHA-type structure ${ }^{31}$ has the spacegroup $R \overline{3} m$ (a squashed cube) with $a=b=c=0.9459 \mathrm{~nm}$, and $\alpha=\beta=\gamma$ $=94.07^{\circ}$. The framework contains double six-membered rings joined together through four-membered rings. The resulting three-dimensional structure has large ellipsoidal chabazite (CHA) cages. Small guest molecules can enter the cages through eight-membered $0.38 \mathrm{~nm}$ wide ring windows. Only linear alkanes are able to penetrate the windows. Each CHA-type unit cell contains a single chabazite cage as shown in Figure $1 \mathrm{~b}$.

B. The dcTST Method. Slow diffusion of molecules in zeolites can be considered an activated process, in which the particles hop from one free-energy minimum to the next, and the actual crossing time is negligible compared to the time a particle spends inside the cage. One can exploit the large separation in time scales using rare-event simulation techniques. ${ }^{36,37}$ We consider a system that can be in two stable states, $\mathrm{A}$ and $\mathrm{B}$, with a dividing free-energy barrier between them. The reaction coordinate $q$ indicates the progress of the diffusion event from cage A to cage $\mathrm{B}$. The location of the dividing barrier is denoted by $q^{*}$. We introduce two characteristic functions $n_{\mathrm{A}}$ and $n_{\mathrm{B}}$ that measure whether the system is in state $\mathrm{A}$ or $\mathrm{B}$. A possible and often used definition is

$$
\begin{aligned}
& n_{\mathrm{A}}=\theta\left(q^{*}-q\right) \\
& n_{\mathrm{B}}=\theta\left(q-q^{*}\right)
\end{aligned}
$$

where $\theta$ is the Heaviside function $\theta(x)$, which has value 0 for $x<0$ and value 1 for $x \geq 0$. 
In the Bennett-Chandler approach ${ }^{26,36,37}$ one computes the hopping rate over the barrier in two steps

$$
\begin{aligned}
k_{\mathrm{A} \rightarrow \mathrm{B}} & =\frac{\left\langle\delta\left(q^{*}-q\right)\right\rangle}{\left\langle\theta\left(q^{*}-q\right)\right\rangle} \\
& \times \frac{\left\langle\dot{q}(0) \delta\left(q^{*}-q(0)\right) \theta\left(q(t)-q^{*}\right)\right\rangle}{\left\langle\delta\left(q^{*}-q(0)\right)\right\rangle} \\
& =\frac{e^{-\beta F\left(q^{*}\right)}}{\int_{\text {cage } \mathrm{A}} e^{-\beta F(q)} \mathrm{d} q} \times\left(\kappa \times \sqrt{\frac{k_{\mathrm{B}} T}{2 \pi m}}\right)
\end{aligned}
$$

where $\delta$ is the Dirac delta function, $q(t)$ the reaction coordinate at time $t, m$ is the mass of the segments of the particle involved in the reaction coordinate, $\kappa$ the transmission coefficient, $k_{\mathrm{B}}$ the Boltzmann constant, $T$ the temperature, $\beta=1 / k_{\mathrm{B}} T$ the inverse temperature, and $F$ the free energy of position related to the probability $P(q)$ by $\beta F=-\ln \langle P(q)\rangle$.

O. Braun and C. Sholl ${ }^{38}$ developed a technique to calculate the diffusion tensor for the lattice-gas model at infinite dilution for any complex elementary cell. Although sometimes lengthy calculations are involved, in many cases analytical expressions for the diffusion tensor may be obtained. It is important to note that the extension to higher loading requires an estimate of the correlations between the particles, which tends to reduce the diffusion. Transition state theory methods such as the methods of Tunca and Ford ${ }^{39-41}$ neglect the correlations while computing the hopping rate, but hope to regain these correlations again during a coarse-grained kinetic Monte Carlo simulation. In addition, various approximations are made to make the computation tractable using multidimensional TST. Their method underpredicted the diffusivity at low loading, while significantly overpredicting the diffusivities at higher loadings, in comparison to conventional MD (see Figure 6 of Ref 41) for methane in LTA-type silica.

The newly developed method of Beerdsen et al. ${ }^{29}$ computes an effective hopping, i.e., a hopping rate including correlations. The conversion of the hopping rate at nonzero loading is therefore the same as for the infinite dilution case. Importantly, the study of diffusion over long time and space regions can then be restricted to the analysis of the free energy profiles of a single unit cell (the surrounding cages influence this profile and have to be properly modeled ${ }^{29}$ ). For the same system, methane in LTA-type silica, the dcTST method of Beerdsen et al. gives exact overlap with MD results (see Figure 3 of ref 29).

For systems with erratic free-energy landscapes, e.g., multiple of barriers of different heights, the dcTST method can be generalized using

$$
k_{\mathrm{A} \rightarrow \mathrm{B}}=\frac{1}{\left\langle n_{\mathrm{A}}\right\rangle} \frac{\left\langle\int_{0}^{\infty} \dot{q}(t) \dot{q}(0) \frac{w(q(t))}{w(q(0))} \frac{e^{-\beta F(q(t))}}{e^{-\beta F(q(0))}} \mathrm{d} t\right\rangle_{\pi}}{\int_{q_{\mathrm{A}}}^{q_{\mathrm{B}}} e^{\beta F(q)} \mathrm{d} q \int_{A+B} e^{-\beta F(q)} \mathrm{d} q}
$$

with a biasing function $w(q)$ operating on the regions $\mathrm{A}$ and $\mathrm{B}$

$$
\begin{gathered}
w(q)=e^{a \beta F(q)} \\
\pi(q) \propto e^{(a-1) \beta F(q)}
\end{gathered}
$$

where $a>0$ is an integer to be chosen freely. A value of $a=$ 1 would flatten the free-energy landscape, a value of $a=2$ would reproduce the Ruiz-Montero method (if the approximate free energy is taken to be the true free energy). ${ }^{42}$ Starting configurations at the desired loading are sampled using NVTMC (with the biased, tagged particle in the $\pi$-ensemble), and subsequently a weighted velocity autocorrelation of the tagged particle is computed using conventional NVE-MD. For more details on the dcTST-methods see refs 26 and 29, and see ref 43 for another application on MFI-type zeolite.

C. Nonrectangular Unit Cells and Reaction Coordinates. ERI and CHA-type zeolites can be described both in terms of rectangular unit cells and in their crystallographic nonrectangular unit cell definitions. In crystallography, the crystal structure is defined by the unit cell, and by the fractional coordinates of the atoms within the unit cell. These coordinates form an orthonormal dimensionless $\mathcal{S}$ space. $\mathcal{S}$ space is often more convenient for the computation of the free-energy profiles. The transformation from $\mathcal{S}$ space to real $\mathscr{R}$ space can be carried out by the matrix $\mathscr{K}$ :

$$
\mathscr{H}=\left(\begin{array}{lll}
a & b \cos (\gamma) & c \cos (\beta) \\
0 & b \sin (\gamma) & c \zeta \\
0 & 0 & c \sqrt{1-\cos ^{2} \beta-\xi^{2}}
\end{array}\right)
$$

with

$$
\zeta=\frac{\cos \alpha-\cos \gamma \cos \beta}{\sin \gamma}
$$

Conversely, $\mathscr{K}^{-1}$ transforms real coordinates into fractional coordinates. With the chosen $\mathscr{K}$ the scaled box has a length of 1. Our potential force field is defined in real space, therefore it is convenient to store positions in $\mathscr{R}$ space, transform them to $\mathcal{S}$ space, apply periodic boundary conditions in $\int$ space, and transform back to $\mathscr{R}$ space to compute distances within the simulation box

$$
\begin{gathered}
\mathbf{s}=\mathscr{H}^{-1} \mathbf{r} \\
\mathbf{s}^{\prime}=\mathbf{s}-\operatorname{rint}(\mathrm{s}) \\
\mathbf{r}^{\prime}=\mathscr{H} \mathbf{s}^{\prime}
\end{gathered}
$$

where the "rint" function returns the rounded integer value of its argument. The smallest perpendicular width of the unit cell has to be larger than twice the spherical cutoff in $\mathscr{R}$ space.

For computational reasons, a rectangular unit cell is preferred. Not only is the matrix conversion expensive, if the unit cell is severely distorted from cubic, many redundant distance calculations will be performed for particles lying outside the cutoff in $R$ space, further reducing the efficiency. However, for computation of free-energy profiles in complex zeolite structures, the fractional space is often very convenient.

We can choose $q$ as the position of one of the two beads of ethane. ${ }^{6,7}$ This choice of order parameter underestimates the free energy of the true transition state, but the dynamical correction $\kappa$ is the exact correction compensating our choice of reaction coordinate. ${ }^{37}$

The monoclinic unit cell definition for an ERI-type cage is shown in Figure 1a. Because two cages are present in the unit cell definition it is convenient to be able to select a single cage by using

$$
q= \begin{cases}s_{x}+\left(1-s_{y}\right)<1 & \text { for cage A } \\ s_{x}+\left(1-s_{y}\right)>1 & \text { for cage B }\end{cases}
$$

where $s$ are the fractional coordinates of a single ERI-type unit cell in $\int$ space. For $x y$ computations the barrier is located at $s_{x y}$ $=\{1 / 2,1 / 2\}$, and by symmetry all positions in the unit cell can 
be mapped on the reaction coordinate. For the $z$ computation we select only cage A and use

$$
q=s_{z}
$$

The barrier at the center of the cage is located at $s=\{1 / 3,2 / 3$, $3 / 4\}$, while the free-energy minima $q^{\mathrm{A}}$ and $q^{\mathrm{B}}$ are located at $s$ $=\{1 / 3,2 / 3,1 / 2\}$ and $s=\{1 / 3,2 / 3,1\}$, respectively. Using the projection eq 11 and the positions of cage A only, $q^{\mathrm{A}}$ is located at $s_{z}=1 / 2$, the barrier $q^{*}$ at $s_{z}=3 / 4$, and $q^{\mathrm{B}}$ at $s_{z}=1$.

The unit-cell definition for a CHA-type cage is shown in Figure $1 b$ (the rhombohedral lattice can be thought of as a cube slightly pulled along its space diagonal). The reaction coordinate can now be chosen from the center of the cage $\left(\mathbf{s}_{0}=\{0.5,0.5\right.$, $0.5\})$ to any of the six exits through the center of the windows $\left(\mathbf{s}_{1}=\{1,0.5,0.5\}, \mathbf{s}_{2}=\{0.5,1,0.5\}, \mathbf{s}_{3}=\{0.5,0.5,1\}, \mathbf{s}_{4}=\right.$ $\left.\{0,0.5,0.5\}, \mathbf{s}_{5}=\{0.5,0,0.5\}, \mathbf{s}_{6}=\{0.5,0.5,0\}\right)$, i.e., the space is simply mapped onto the three orthonormal axes in scaled space. The other half of the profile, i.e., from $q^{*}$ to $q^{B}$ follows by symmetry. For symmetry reasons, the free-energy profiles are all equivalent, and there is only one hopping rate $k$ from a cage to any of the neighboring cages in CHA-type zeolites.

D. Force Field Potentials and Simulation Details. We neglect cations and study rigid, all-silica versions of the ERIand CHA-type zeolites. The positions of the atoms are taken from refs 30 and 31, respectively. Following the work of Bezus et al., ${ }^{44}$ the zeolites are modeled as a rigid network of oxygen and silicon atoms. This is a very common approximation because lattice flexibility is not that important for small alkanes in all-silica zeolites. ${ }^{45,46}$ The simulation box sizes we used are $3 \times 3 \times 3$ (perpendicular widths are $3.448 \times 3.448 \times 4.515$ nm) for ERI-type zeolite, and $3 \times 3 \times 3$ (perpendicular widths are $2.81 \times 2.81 \times 2.81 \mathrm{~nm}$ ) for CHA-type zeolite. Tests on larger systems did not show any significant finite-size effects. Periodic boundary conditions were employed. Adsorption in cation-free structures takes place at sites with little or no electric field. For these reasons the united atom model ${ }^{47}$ seems the most straightforward choice. We consider the $\mathrm{CH}_{3}$ groups as single, chargeless interaction centers with their own effective potentials. The beads of ethane are connected by an harmonic bonding potential $U^{\text {bond }}=1 / 2 k_{1}\left(r-r_{0}\right)^{2}$ with $k_{1} / k_{\mathrm{B}}=96500 \mathrm{~K} / \AA^{2}$ and $r_{0}=1.54 \AA$. The extramolecular energy $U^{\text {ext }}$ consists of a guest-guest intermolecular energy $U^{\text {gg }}$, a host-guest interaction $U^{\text {hg }}$, modeled with a Lennard-Jones potential with a cutoff radius of $12 \AA$. The parameters $\sigma_{\mathrm{O}-\mathrm{CH}_{3}}=3.17 \AA, \epsilon_{\mathrm{O}-\mathrm{CH}_{3}} / k_{\mathrm{B}}=142 \mathrm{~K}$, $\sigma_{\mathrm{Si}-\mathrm{CH}_{3}}=2.12 \AA, \epsilon_{\mathrm{Si}-\mathrm{CH}_{3}} / k_{\mathrm{B}}=82 \mathrm{~K}$, and $\sigma_{\mathrm{CH}_{3}-\mathrm{CH}_{3}}=3.78 \AA$, $\epsilon_{\mathrm{CH}_{3}-\mathrm{CH}_{3}} / k_{\mathrm{B}}=104 \mathrm{~K}$ were taken from ref 48 . Although the size parameters are rather small, for this study we prefer to use these parameters because then diffusion of ethane in ERI- and CHAtype zeolite is still feasible using conventional MD.

The simulations were performed using two different methods: the recently proposed dynamically corrected transition state theory and conventional molecular dynamics (MD) (dcTST). ${ }^{26,29}$ We used the velocity Verlet integration scheme with a time step of $0.5 \mathrm{fs}$. The relative energy drift was smaller than $10^{-4}$. For temperature control we employed the Nosé-Hoover chain (NHC) method as formulated by Martyna et al. ${ }^{49}$ Molecules were inserted into the framework at random positions as long as no overlaps occurred with the framework or other particles. During the initialization period, we performed an NVT Monte Carlo (MC) simulation to rapidly achieve an equilibrium molecular arrangement. After the initialization period, we assigned velocities from the Maxwell-Boltzmann distribution at the desired average temperature to all the atoms. The total momentum of the system was set to zero. Next, we equilibrated the system further by performing an NVT MD simulation using the NHC thermostat. After the equilibration was completed, during the production run of more than $20 \mathrm{~ns}$, we collected statistics using the NVT-ensemble. Simulations using the NVE-ensemble gave equivalent results. More details can be found in ref 26 .

Transmission coefficients are computed from at least 50000 independent configurations. These configurations are obtained from Monte Carlo simulations, where a configuration is stored every 500 cycles. A cycle is defined as $N$ steps, where $N$ is the number of molecules, and a step is one Monte Carlo move (translation, rotation, full regrow). On average there is one Monte Carlo move per particle in a single cycle. The free-energy profiles are obtained using $\mathrm{MD}$ and $\mathrm{MC}$; both give equivalent results.

\section{Results}

A. Ethane in ERI-type Zeolite (anisotropic diffusion). In an ERI-type lattice, diffusion in the $x y$ plane occurs isotropically on an hexagonal lattice

$$
D_{x y}=\frac{1}{4} k_{x y} \lambda_{x y}^{2}
$$

with $\lambda_{x y}$ the lattice displacement distance and $k_{x y}$ the corresponding hopping rate. The $z$ diffusion is dependent on the hopping in the $x y$ plane. In general, the method of Braun and Sholl ${ }^{38}$ can be used to compute diffusivity tensors. However, for such sequential hops we can derive a simple formula for the combined hopping rate, based on the mean first passage time. Consider a hop from $\mathrm{A}$ to $\mathrm{C}$ with an intermediate state $\mathrm{B}$. We have

$$
\begin{gathered}
\frac{P_{\mathrm{B} \rightarrow \mathrm{A}}}{P_{\mathrm{B} \rightarrow \mathrm{C}}}=\frac{k_{\mathrm{B} \rightarrow \mathrm{A}}}{k_{\mathrm{B} \rightarrow \mathrm{C}}} \\
P_{\mathrm{B} \rightarrow \mathrm{A}}+P_{\mathrm{B} \rightarrow \mathrm{C}}=1
\end{gathered}
$$

and therefore

$$
\begin{aligned}
P_{\mathrm{B} \rightarrow \mathrm{C}} & =P_{\mathrm{B} \rightarrow \mathrm{A}} \frac{k_{\mathrm{B} \rightarrow \mathrm{C}}}{k_{\mathrm{B} \rightarrow \mathrm{A}}} \\
& =\left(1-P_{\mathrm{B} \rightarrow \mathrm{C}}\right) \frac{k_{\mathrm{B} \rightarrow \mathrm{C}}}{k_{\mathrm{B} \rightarrow \mathrm{A}}}
\end{aligned}
$$

from which $P_{\mathrm{B} \rightarrow \mathrm{C}}$ can be solved:

$$
P_{\mathrm{B} \rightarrow \mathrm{C}}=\frac{k_{\mathrm{B} \rightarrow \mathrm{C}}}{k_{\mathrm{B} \rightarrow \mathrm{A}}+k_{\mathrm{B} \rightarrow \mathrm{C}}}
$$

The hopping rate from $\mathrm{A}$ to $\mathrm{C}$ is the hopping rate from $\mathrm{A}$ to $\mathrm{B}$ times the probability $P_{\mathrm{B} \rightarrow \mathrm{C}}$ to go from B to C

$$
k_{\mathrm{A} \rightarrow \mathrm{C}}=k_{\mathrm{A} \rightarrow \mathrm{B}} P_{\mathrm{B} \rightarrow \mathrm{C}}
$$

In general, a serial combination of hops is then described by

$$
k_{\mathrm{A} \rightarrow \mathrm{C}}=\frac{k_{\mathrm{A} \rightarrow \mathrm{B}} k_{\mathrm{B} \rightarrow \mathrm{C}}}{k_{\mathrm{B} \rightarrow \mathrm{A}}+k_{\mathrm{B} \rightarrow \mathrm{C}}}
$$

Equation 19 proves convenient for finding the relation between 

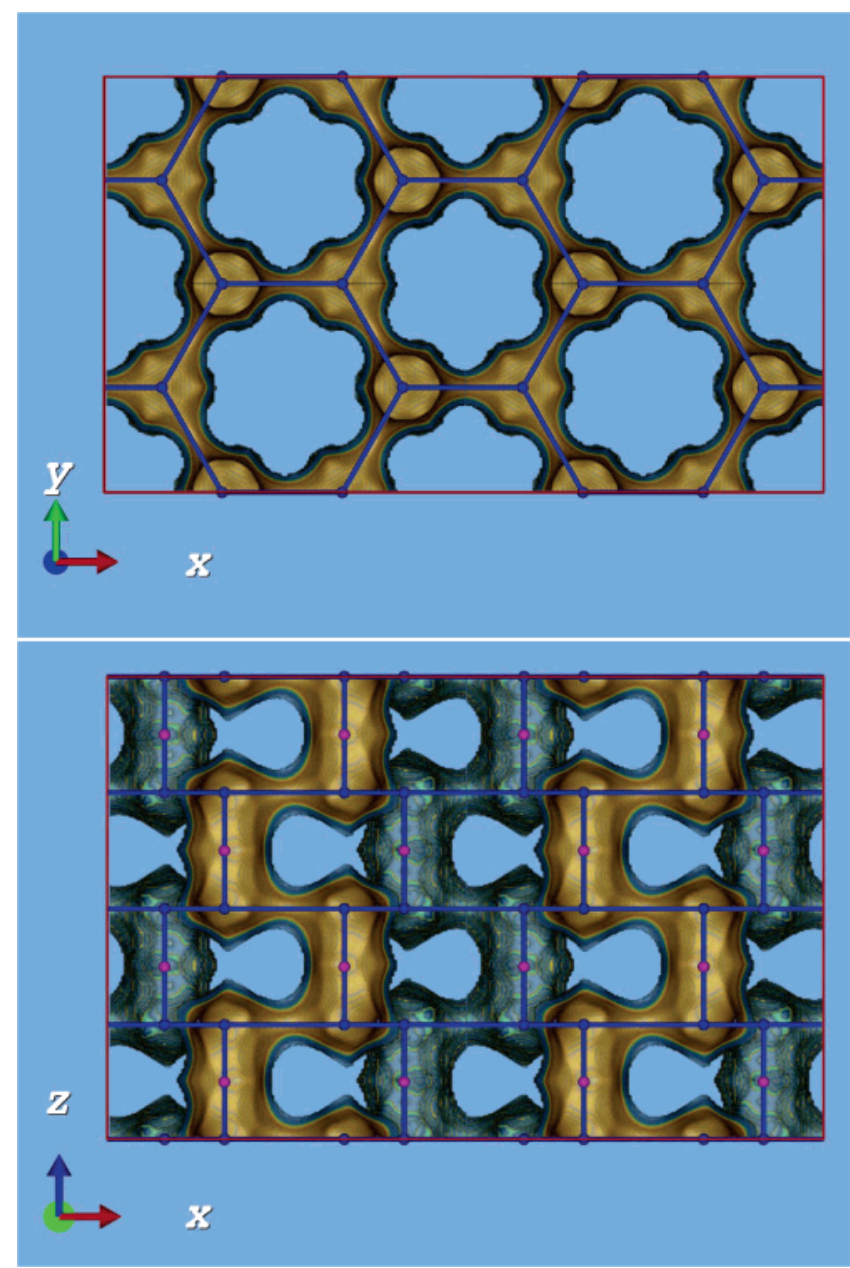

Figure 2. Topology of the ERI-type lattice in (a) $x y$ direction the hopping takes place on a hexagonal lattice, in (b) the $z$ direction a displacement has to be preceded first by a $x y$ hop. The lattice is drawn in blue dots connected by blue lines of lattice distance $\lambda \approx 0.75 \mathrm{~nm}$ for $x, y$, and $z$ directions.

hopping rates and diffusion coefficient on non-Bravais lattices. The relation between $k_{\mathrm{A} \rightarrow \mathrm{B}}$ and $k_{\mathrm{B} \rightarrow \mathrm{A}}$ is given by

$$
\frac{k_{\mathrm{A} \rightarrow \mathrm{B}}}{k_{\mathrm{B} \rightarrow \mathrm{A}}}=\frac{\left\langle n_{\mathrm{B}}\right\rangle}{\left\langle n_{\mathrm{A}}\right\rangle}
$$

where $\left\langle n_{\mathrm{A}}\right\rangle=1-\left\langle n_{\mathrm{B}}\right\rangle$ is the equilibrium mole fraction of particles in state A. For a symmetric barrier $\left\langle n_{\mathrm{A}}\right\rangle=\left\langle n_{\mathrm{B}}\right\rangle$ and $k_{\mathrm{A} \rightarrow \mathrm{B}}=k_{\mathrm{B} \rightarrow \mathrm{A}}$, so

$$
k_{\mathrm{A} \rightarrow \mathrm{C}}=\frac{k_{\mathrm{A} \rightarrow \mathrm{B}} k_{\mathrm{B} \rightarrow \mathrm{C}}}{k_{\mathrm{A} \rightarrow \mathrm{B}}+k_{\mathrm{B} \rightarrow \mathrm{C}}}
$$

The lattice displacement vector $\lambda_{z}$ is orthogonal to $\lambda_{x y}$ and, using eq 21 plus the symmetry of the lattice, we derive immediately

$$
D_{z}=\frac{1}{2} \frac{k_{x y} k_{z}}{k_{x y}+k_{z}} \lambda_{z}^{2}
$$

Diffusion in ERI-type lattices is strongly anisotropic. In Figure 3 we show the measured mean-squared displacements for ethane obtained using MD at $600 \mathrm{~K}$ and a loading of one molecule per cage. Mean-squared displacements (MSDs) are equal for $x$ and $y$ directions and different in the $z$ direction. The units are convenient for simulation purposes, because distances are often defined in angstroms, and the relevant time scale is in the

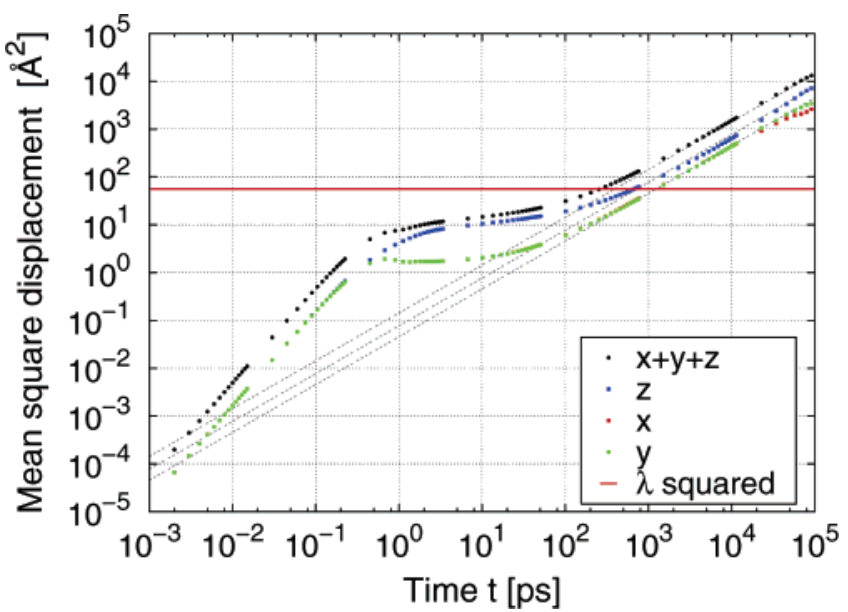

Figure 3. Mean-squared displacement of ethane in ERI-type zeolite at $600 \mathrm{~K}$ and a loading of one molecule per cage. The MSD is equal for $x$ and $y$ directions, and different in the $z$ direction. The horizontal line is the cage-to-cage hopping distance $\lambda$ (approximately $7.5 \AA$ for both the $x y$ and $z$ directions). The different regimes of diffusion are clearly visible, and only after a single cage-crossing has the MSD become linear (a straight line of slope one in $\log -\log$ scale). The slope over the linear regime corresponds to the limit of long times and can then be reliably measured. The diffusion coefficient is the slope at long times divided by two times the number of spatial dimensions.

picosecond range. Slopes of MSDs are therefore in units of 1 $\times 10^{-8} \mathrm{~m}^{2} / \mathrm{s}$. The different regimes of diffusion are clearly visible, and only after a single cage-crossing the MSD has become linear (a straight line of slope one in $\log -\log$ scale). For interacting particles, particle-particle and particle-zeolite collisions occur on a different time-scale. The mean-squared displacement thus bends over to attain a different slope. We are interested in the long-time diffusion coefficient. The selfdiffusion coefficients $D_{\alpha}$ in the direction $\alpha=x, y, z$ are computed by taking the slope at long times

$$
D_{\alpha}=\frac{1}{2 N} \lim _{t \rightarrow \infty}-\left\langle\sum_{i=1}^{N}\left(r_{i \alpha}(t)-r_{i \alpha}(0)\right)^{2}\right\rangle
$$

where $N$ is the number of molecules, $t$ the time, and $r_{i \alpha}$ the $\alpha$-component of the center-of-mass of molecule $i$. The diffusion coefficients at one molecule per cage $600 \mathrm{~K}$ are $D_{x y}=2.1 \times$ $10^{-9} \mathrm{~m}^{2} / \mathrm{s}$ and $D_{z}=4.2 \times 10^{-9} \mathrm{~m}^{2} / \mathrm{s}$. This indicates the diffusion is a rare event and the windows form obstructions to diffusion. Because $D_{z} \approx 2 D_{x y}$ there are apparently no significant freeenergy barriers inside an erionite cage at low loading.

The hopping lattice for most cage/window-type zeolites is formed by the lattice spanned by their cage centers. However, for elongated cages as in erionite, intracage barriers are formed at higher loadings. For an analysis we measure the free-energy profiles along the cage-length (the $z$ direction), and along the center-to-center line in the hexagonal $x y$ plane. The profiles of ethane plotted in Figure 4 over various loadings indicate that indeed there are internal cage barriers, and for the $x y$ plane the barrier is formed by the eight-ring dividing window. The diffusion coefficient in the $z$ direction depends on both the hopping rate in the $z$ direction and the $x y$ direction (eq 22), because each hop in the $z$ direction has to be preceded by a hop in the $x y$ plane.

The free-energy barrier in the $x y$ plane is sharply peaked, and therefore the transmission coefficient is straightforward to evaluate using the Bennet-Chandler approach. The transmission coefficients $\kappa(t)$ are shown in Figure 5. The starting configurations, with the reaction coordinate constrained to the top of the 


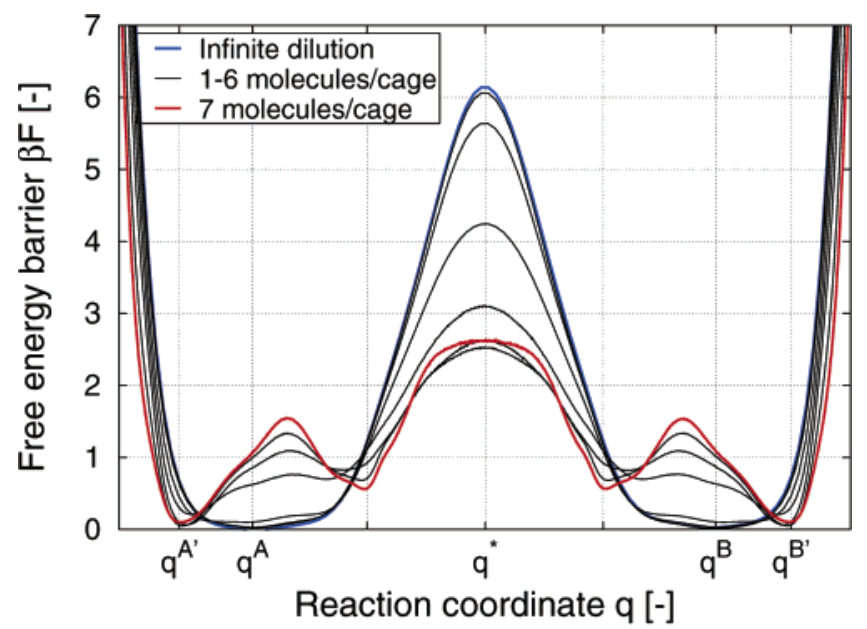

(a)

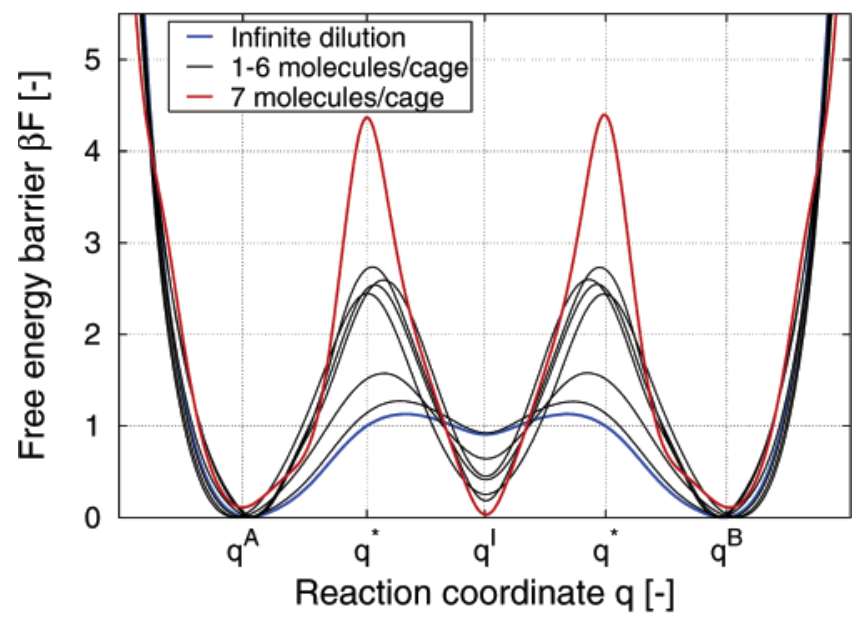

(b)

Figure 4. Free energy profiles $F(q)$ at $600 \mathrm{~K}$ of ethane in ERI at various loadings (infinite dilution, 1, 2, 3, 4, 5, 6, and 7 molecules per erionite cage) in the (a) hexagonal $x y$ plane with $q_{\mathrm{A}}$ the center of a cage, and $q_{\mathrm{B}}$ the center of a neighboring cage, (b) in the $z$ direction across a cage with $q_{\mathrm{A}}$ the top of the cage, $q_{\mathrm{I}}$ the middle of the cage, and $q_{\mathrm{B}}$ the bottom of the cage, respectively.

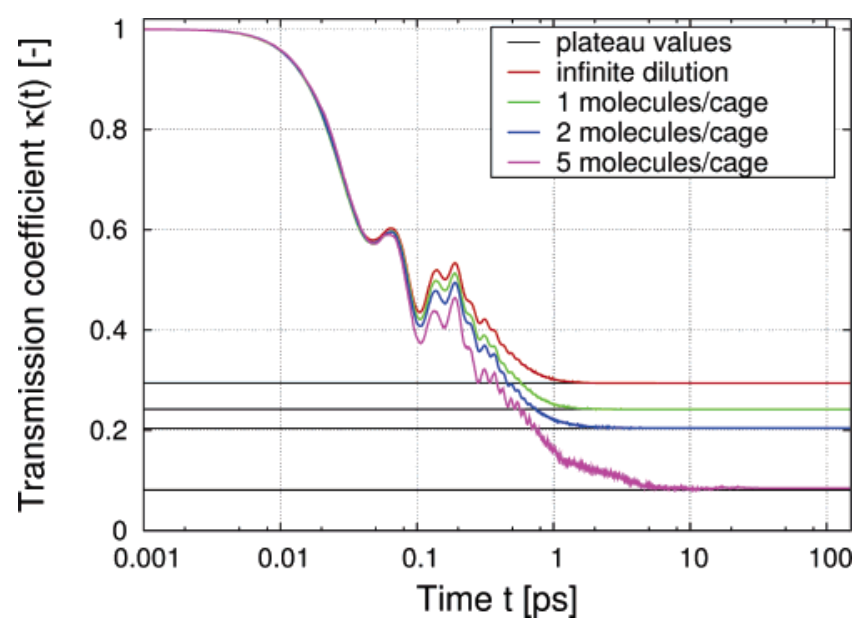

Figure 5. Transmission coefficient $\kappa(t)$ for ethane at $600 \mathrm{~K}$ in ERItype zeolite as a function of time for various loadings.

barrier $q^{*}$, were sampled using a Monte Carlo scheme. After approximately 10 picoseconds the time-dependent transmission coefficient $\kappa(t)$ reached its plateau value $\kappa$. Using eq 4 and eq

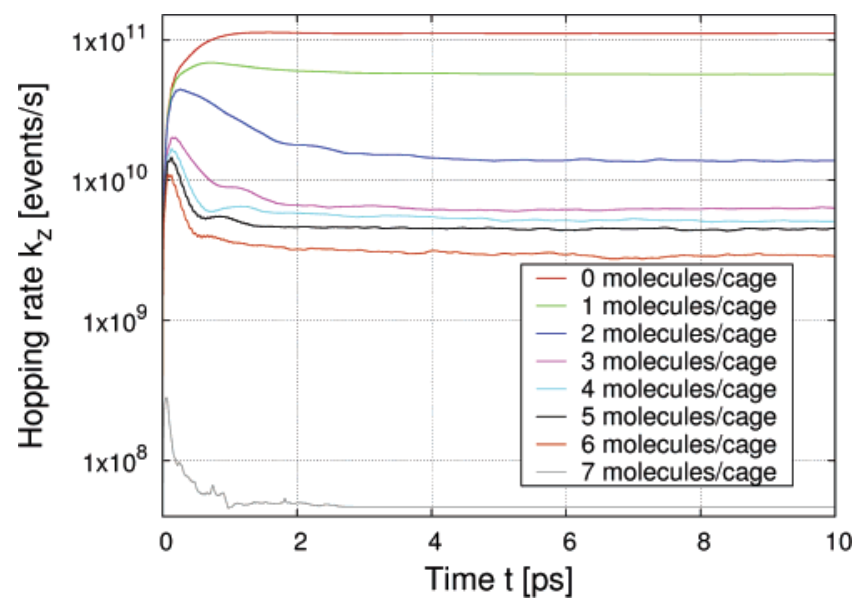

Figure 6. Intracage hopping rate in the $z$ direction obtained using biased MD for ethane in ERI-type cages at $600 \mathrm{~K}$.

12 the hopping rate and diffusion coefficient in the $x y$ plane can be computed.

The free energy in the $z$ direction across an erionite cage is initially rather flat, and with increasing loading a clear freeenergy minimum is formed in the center of the cage. There are two solutions to this problem. The first would be to use a different hopping lattice and include the pink points in Figure 2 in the hopping lattice. A hop in the $z$ direction is now a sequential process of two hops, and the total hopping rate can be obtained using eq 21 . However, when there are several hopping rates very different in magnitude, it is more complicated to define a hopping rate for the fast jumping particles, because kinetic correlations are abundant.

A second method, and the method of our choice here, is to use biased MD to compute the total hopping rate from the top of the cage to the bottom of the cage directly. The reasons are two-fold: first the method is applicable to low free-energy barriers, and second, the method is able to compute hopping rates over complicated free-energy landscapes such as, in this case, two barriers. The reaction coordinate is the projection on the $z$ axis (eq 11), where $q_{\mathrm{A}}$ denotes the top of the cage, $q_{\mathrm{B}}$ the bottom of the cage, and $q_{\mathrm{I}}$ the intermediate free-energy minimum. There are two barriers, one separating $q_{\mathrm{A}}$ and $q_{\mathrm{I}}$, and another one between $q_{\mathrm{I}}$ and $q_{\mathrm{B}}$. The biased MD method computes the total hopping rate from $q_{\mathrm{A}}$ to $q_{\mathrm{B}}$ by computing an effective diffusion coefficient over the entire $q$ domain $\left(q_{\mathrm{A}}\right.$ to $\left.q_{\mathrm{B}}\right)$, i.e., there is no separate computation of the transmission coefficient. The results are shown in Figure 6. The plateau value at long times is the hopping rate of interest.

The self-diffusion coefficients of ethane in ERI-type zeolite at $600 \mathrm{~K}$ using dcTST and conventional MD are shown in Figure 7. Surprisingly, the anisotropical behavior of ERI-type cages reverses with loading, i.e., at low loading the diffusion in the $z$-direction is two times faster than in the $x y$ direction, while for higher loadings this changes to a diffusion that is more than two times slower. Although MD and dcTST give equivalent diffusivity results, the behavior is better understood by analyzing the free-energy profiles (and transmission coefficients). At low loading the diffusion is impeded by the eight-ring windows, i.e., the exits out of the cage to the next, but at higher loadings the barrier is formed by the center of the cages.

B. Ethane in CHA-type Zeolite. We note that the same formulas have been derived before, but for CHA-type zeolites by Schüring et al. ${ }^{35}$ Examining Figure 8, it appears that CHAand ERI-type zeolites are very similar, the difference being the more elongated shape of ERI-type cages. In such a lattice one 


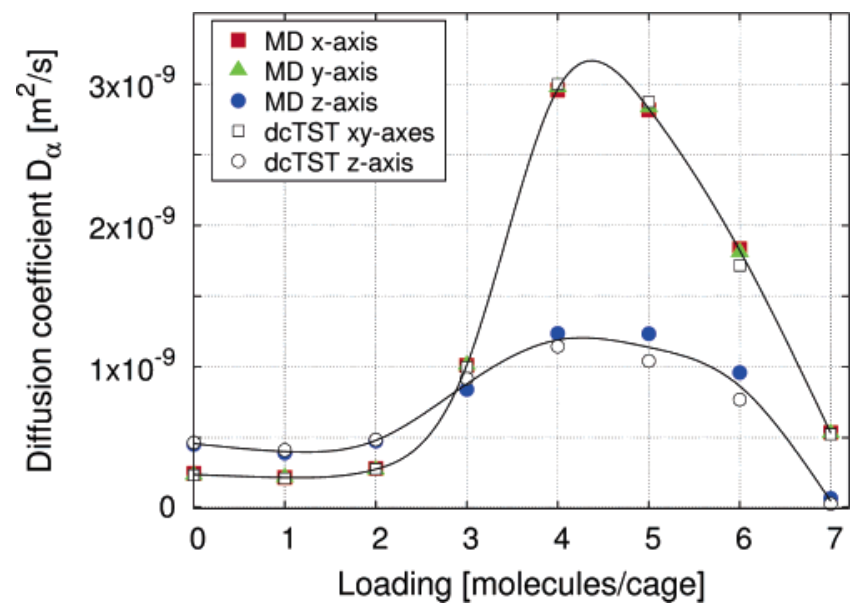

Figure 7. Anisotropic diffusion of ethane in ERI-type zeolite computed by dcTST and conventional MD at $600 \mathrm{~K}$.

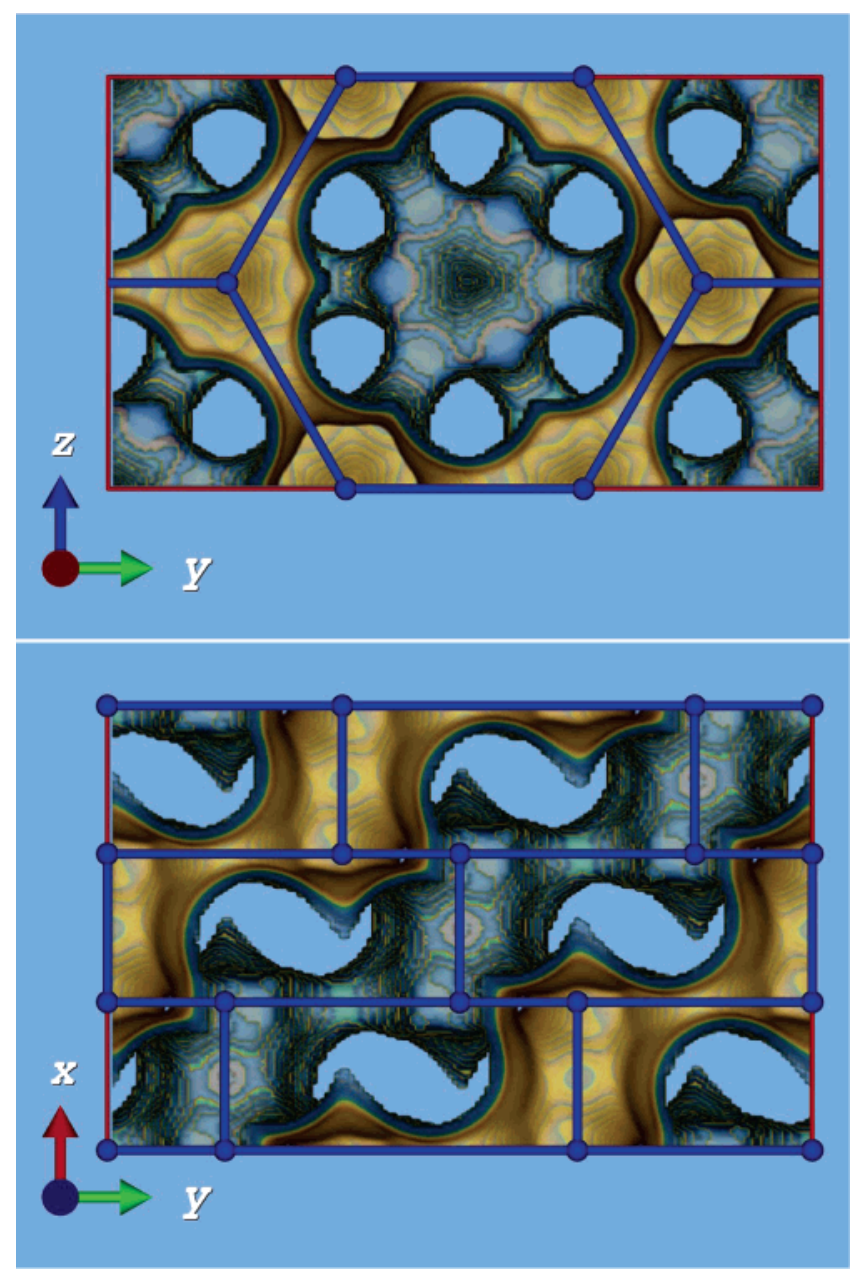

Figure 8. A possible rectangular topology of the CHA-type lattice used in the literature. ${ }^{34,35}$ In the $z y$ direction (a), the hopping takes place on a hexagonal lattice; in the $x$ direction (b), a displacement has to be preceded first by a $z y$ hop. The lattice is drawn in blue dots connected by blue lines.

expects the diffusion to be anisotropic, and this is indeed the case as evidenced by the mean-squared displacements in Figure 10. To convert the hopping rate in CHA-type zeolites to a diffusion coefficient, we note that the lattice is actually only slightly distorted from a cubic lattice (Figure 9). The orientationally averaged diffusion coefficient is not affected by the distortion in CHA-type lattices but the individual components

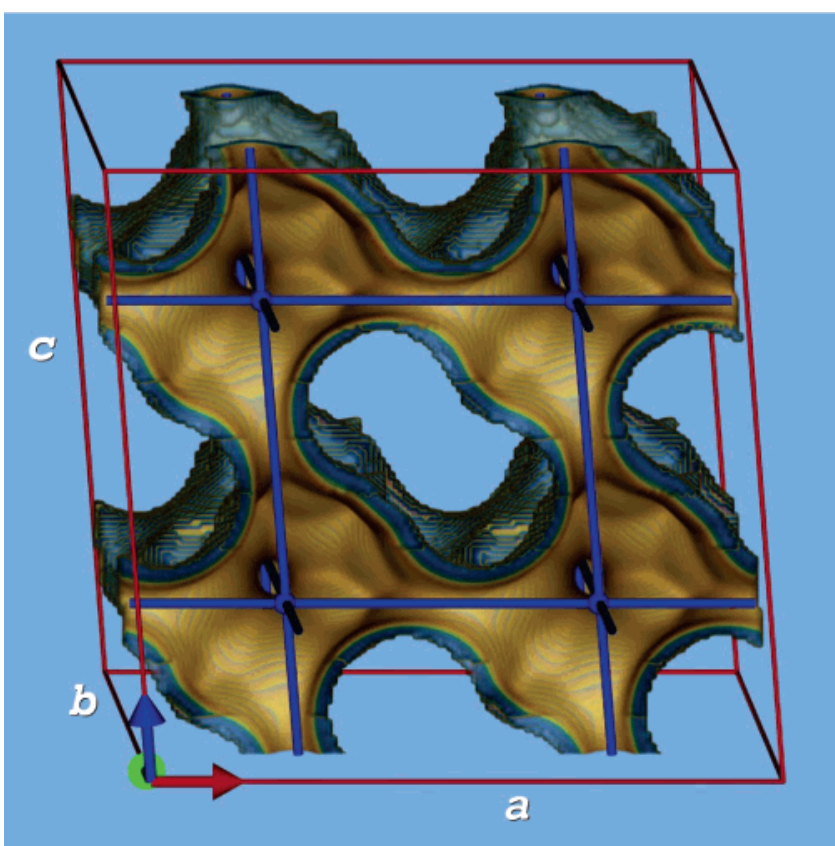

Figure 9. "Squashed cube" topology of the CHA-type lattice. The lattice is drawn in blue dots connected by blue lines of lattice distance $\lambda \approx 0.9459 \mathrm{~nm}$ for $a, b$, and $c$ directions.

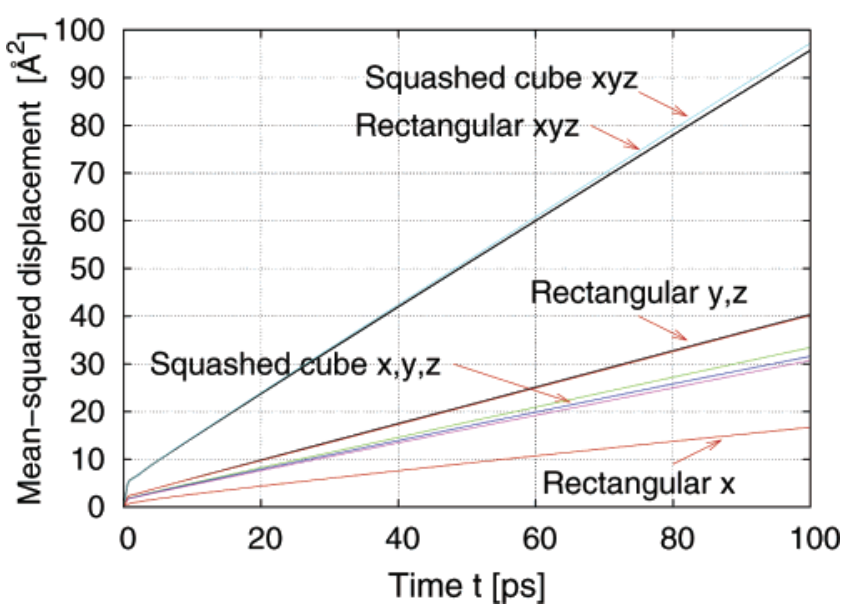

Figure 10. Mean-squared displacements of ethane at $600 \mathrm{~K}$ and a loading of one molecule per cage for the rectangular and "squashed cube" version of CHA-type zeolites.

are. However, the distortion effect for the CHA-type lattice is negligibly small (smaller than 2\%). Therefore, diffusion in CHAtype zeolite can be considered isotropic in practice using this lattice (see Figure 10). CHA-type zeolites only appear to be anisotropic when the lattice is not properly aligned with the crystal axes. We note that, unlike in lattice theory, in our systems both the lattice and the hopping rates need to be found.

A similar free energy analysis as for ERI-type zeolites can be performed for CHA-type zeolites. However, for the "squashed cube", the free-energy profiles and transmission rates are equal for $a, b$, and $c$ directions. Hence, there is only one hopping rate, and any anisotropy is entirely due to the small distortion from a perfect cube. In Figure 11 we show the free-energy profiles for CHA-type zeolites. The barrier is formed by the eight-membered ring for all loadings, and at higher loadings some intracage reorganization is observed as more and more molecules have to be accommodated inside the chabazite cage. 


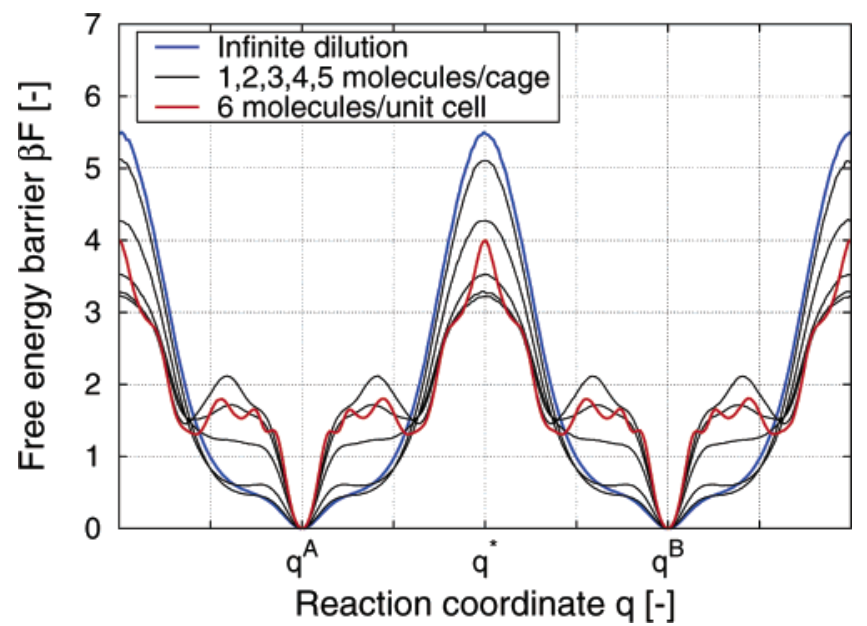

Figure 11. Free energy profiles $F(q)$ at $600 \mathrm{~K}$ of ethane in CHA at various loadings (infinite dilution, 1, 2, 3, 4, 5, and 6 molecules per chabazite cage). The reaction coordinate is the position of the first bead along the centerline connecting the center points of two cages (lines from top to bottom in order of the legend).

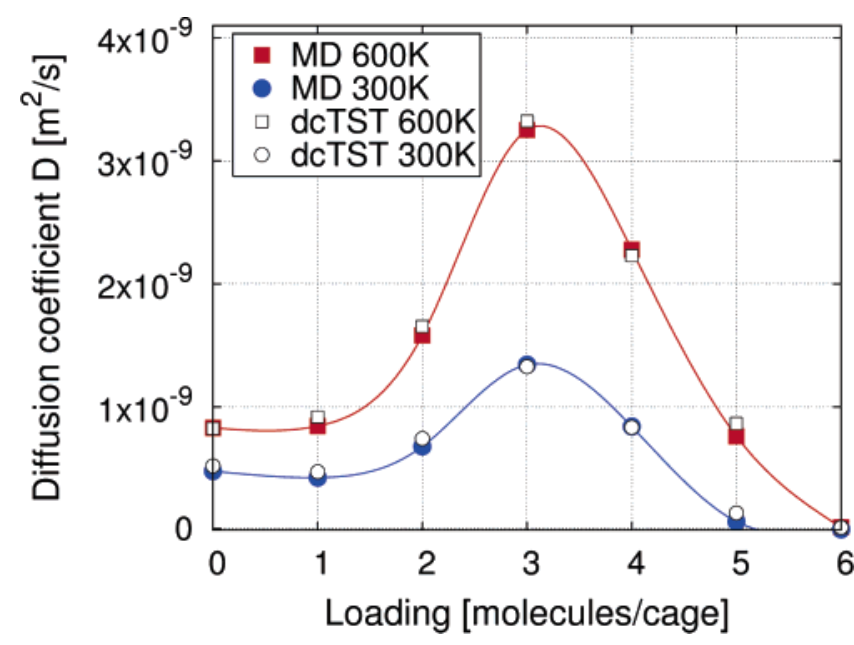

Figure 12. Orientationally averaged diffusion of ethane in CHA-type zeolite computed by dcTST and conventional MD at $300 \mathrm{~K}$ and 600 K.

Our simulations indicate no significant anisotropy (less than $2 \%$ ), and therefore we plot the orientationally averaged selfdiffusivity only for both MD and dcTST in Figure 12. A similar diffusivity behavior to ERI-type zeolites is observed for CHAtype cages. Indeed, the increase in diffusivity is a general feature present due to cage confinement. The maximum in the diffusivity is shifted to lower loading, consistent with the smaller cage size of chabazite in comparison to erionite.

As mentioned previously, the diffusion in CHA-type zeolite can be considered isotropic in practice. Indeed, tracer-diffusion measurements of water in natural chabazite by Raman spectroscopy did not indicate any substantial deviation from diffusion isotropy. ${ }^{50}$ However, using the pulsed field gradient NMR technique, Bär et al. ${ }^{34}$ reported an orientation-dependent diffusivity with a ratio between the maximum and minimum diffusivity of a factor of two, also for water in natural chabazite. Such a significant anisotropy could potentially originate from a significant symmetry breaking in the zeolite sample, caused for instance by a nonrandom arrangement of cations and/or imperfections inside the crystal.

\section{Conclusions}

The dcTST gives results equivalent to conventional MD, but is also applicable in the regime of very slow diffusion where MD cannot be used. Moreover, the method allows for a more detailed analysis in terms of free-energy profiles and transmission coefficients. The first is a static term, corresponding to locations of preferable adsorption sites and estimates of freeenergy barriers in between, the latter (or actually the inverse of the transmission coefficient: the recrossing) corresponds to collision frequencies, which generally increase with loading. Here, we have shown how to apply the dcTST to nontrivial lattices: (a) ERI-type lattices are non-Bravais, (b) CHA-type lattices are rhombohedral. Both zeolites are cage/window-type zeolites, and in both zeolites the diffusion increases with loading and decreases only close to saturation loading. The diffusion of CHA-type zeolites is nearly isotropic, the diffusion of ERItype zeolites is strongly anisotropic. Surprisingly, the anisotropic behavior of ERI-type cages reverses with loading, i.e., at low loading the diffusion in the $z$ direction is two times faster than in the $x y$ direction, while for higher loadings this changes to a diffusion that is more than two times slower. The computation using TST for ERI-type zeolites required the combination of two methods: dcTST for the $x y$ plane, and biased MD to compute the much faster intracage hopping rates. Although MD and dcTST give equivalent diffusivity results, the behavior is better understood by analyzing the free-energy profiles (and transmission coefficients). At low loading the diffusion is impeded by the eight-ring windows, i.e., the exits out of the cage to the next, but at higher loadings the barrier is formed by the centers of the cages.

Acknowledgment. We thank T. J. H. Vlugt and T. S. van Erp for their comments on the manuscript, The Netherlands Research Council (CW), the Deutsche Forschungsgemeinschaft (DFG, priority program SPP 1155), and the Spanish Ministry of Science and Technology (Ramón y Cajal Program and VEM2003 20574 C03 01) for financial support, and NWO/NCF for computational resources.

\section{References and Notes}

(1) Gupta, V.; Nivarthi, S. S.; McCormick, A. V.; Davis, H. T. Chem. Phys. Lett. 1995, 247, 596-600.

(2) Kukla, V.; Kornatowski, J.; Demuth, D.; Girnus, I.; Pfeifer, H. Rees, L. V. C.; Schunk, S.; Unger, K. K.; Kärger, J. Science 1996, 272, 702-704. 765.

(3) Hahn, K.; Kärger, J.; Kukla, V. Phys. Rev. Lett. 1996, 76, $2762-$

(4) Gorring, R. L. J. Catal. 1993, 31, 13-26.

(5) Yoo, K.; Tsekov, R.; Smirniotus, P. G. J. Phys. Chem. B 2003, 7 , 13593-13596.

(6) Dubbeldam, D.; Calero, S.; Maesen, T. L. M.; Smit, B. Phys. Rev. Lett. 2003, 90, 245901. 12152

(7) Dubbeldam, D.; Smit, B. J. Phys. Chem. B 2003, 107, 12138-

(8) Ghorai, P. K.; Yashonath, S.; Demontis, P.; Suffritti, G. B. J. Am. Chem. Soc. 2003, 125, 7116-7123.

(9) June, R. L.; Bell, A. T.; Theodorou, D. N. J. Phys. Chem. 1990, 94, 8232-8240.

(10) Kärger, J. J. Phys. Chem. 1991, 95, 5558-5560.

(11) Hong, U.; Kärger, J.; Kramer, R.; Pfeifer, H.; Seiffert, G.; Muller, U.; Unger, K. K.; Luck, H. B.; Ito, T. Zeolites 1991, 11, 816-821.

(12) June, R. L.; Bell, A. T.; Theodorou, D. N. J. Phys. Chem. 1992, 96, 1051-1060

(13) Maginn, E. J.; Bell, A. T.; Theodorou, D. N. J. Phys. Chem. 1996 $100,7155-7173$.

(14) Bussai, C.; Fritzsche, S.; Haberlandt, R.; Hannongbua, S. J. Phys. Chem. B 2003, 107, 12444-12450.

(15) Skoulidas, A. I.; Sholl, D. S. J. Phys. Chem. B 2002, 106, 50585067.

(16) Skoulidas, A. I.; Sholl, D. S. J. Phys. Chem. A 2003, 107, 1013210141. 
(17) Trinh, S.; Locke, B. R.; Arce, P. Transport Porous Med. 2002, 47, 279-293.

(18) Su, Z.; Cushman, J. H.; Currya, J. E. J. Phys. Chem. B 2002, 118 , $1417-1422$.

(19) Powles, J. G.; Murad, S.; Ravi, P. V. Chem. Phys. Lett. 1992, 188 , 21-24. 35

(21) Wingen, A.; Basler, W.; Lechert, H. Stud. Surf. Sci. Catal. 1997 $105,495-500$.

(22) Nelson, P. H.; Tsapatsis, M.; Auerbach, S. M. J. Membr. Sci. 2001 $184,245-255$.

(23) Nelson, P. H.; Auerbach, S. M. Chem. Eng. J. 1999, 74, 43-56.

(24) Furo, I.; Dvinskikh, S. V. Magn. Reson. Chem. 2002, 40, S3S14.

(25) Menzel, M. I.; Han, S.; Stapf, S.; Blümich, B. J. Magn. Reson. 2000, 143, 376-381.

(26) Dubbeldam, D.; Beerdsen, E.; Vlugt, T. J. H.; Smit, B. J. Chem. Phys. 2005, 122, 224712.

(27) Voter, A. F.; Montalenti, F.; Germann, T. C. Annu. Rev. Mater. Res. 2002, 32, 321-346.

(28) Auerbach, S. M. Int. Rev. Phys. Chem. 2000, 19, 155-198.

(29) Beerdsen, E.; Smit, B.; Dubbeldam, D. Phys. Rev. Lett. 2004, 93 , 248301.

(30) Gard, J. A.; Tait, J. M., Proceedings of the Third International Conference on Molecular Sieves; Recent Progress Reports; Uytterhoeven, J. B., Ed.; Leuven University Press: Zurich, 1973; pp 94-99.

(31) Calligaris, M.; Nardin, G.; Randaccio, L. Zeolites 1983, 3, 205208.

(32) Dubbeldam, D.; Beerdsen, E.; Calero, S.; Smit, B. Proc. Natl. Acad. Sci. U.S.A. 2005, 102, 12317-12320.

(33) Clark, L. A.; Ye, G. T.; Snurr, R. Q. Phys. Rev. Lett. 2000, 84, 2893-2896.
(34) Bär, N.-K.; Kärger, J.; Pfeifer, H.; Schäfer, H.; Schmitz, W Microporous Mesopourou Mater. 1998, 22, 289-295.

(35) Schüring, A.; Auerbach, S. M.; Fritzsche, S.; Haberlandt, R. In Random Walk Treatment of Dumb-Bell Molecules in an LTA Zeolite and in Chabazite; Proceedings of the 14th International Zeolite Conference; van Steen, E., Callanan, L., Claeys, M., Eds.; Elsevier: Cape Town, 2004, pp 2110-2117.

(36) Bennett, C. H. In Diffusion in Solids: Recent Developments; Nowick, A. S., Burton, J. J., Eds.; Academic Press: New York, 1975; pp $73-113$.

(37) Chandler, D. J. Chem. Phys. 1978, 68, 2959.

(38) Braun, O. M.; Sholl, C. A. Phys. Rev. B 1998, 58, 14870-14879.

(39) Tunca, C.; Ford, D. M. J. Chem. Phys. 1999, 111, 2751-2760.

(40) Tunca, C.; Ford, D. M. J. Phys. Chem. B 2002, 106, 10982-10990.

(41) Tunca, C.; Ford, D. M. Chem. Eng. Sci. 2003, 58, 3373-3383.

(42) Ruiz-Montero, M. J.; Frenkel, D.; Brey, J. J. Mol. Phys. 1996, 90, 925-941.

(43) Beerdsen, E.; Dubbeldam, D.; Smit, B. Phys. Rev. Lett. 2005, 95 164505 .

(44) Bezus, A. G.; Kiselev, A. V.; Lopatkin, A. A.; Du, P. Q. J. J. Chem. Soc., Faraday Trans. 2 1978, 74, 367-379.

(45) Vlugt, T. J. H.; Schenk, M. J. Phys. Chem. B 2002, 106, 1275712763

(46) F, F. Leroy; Rousseau, B.; Fuchs, A. H. Phys. Chem. Chem. Phys. 2004, 6, 775-783.

(47) Ryckaert, J. P.; Bellemans, A. Faraday Dicuss. Chem. Soc. 1978 $66,95-106$

(48) Schüring, A.; Auerbach, S. M.; Fritzsche, S.; Haberlandt, R. J. Chem. Phys. 2002, 116, 10890-10894.

(49) Martyna, G. J.; Tuckerman, M.; L., D. J. Tobias M.; Klein, Mol. Phys. 1996, 87, 1117-1157.

(50) Goryainov, S. V.; Belitsky, I. A. Phys. Chem. Miner. 1995, 22, $433-452$. 